\title{
Elevated Plasma Homocysteine Level in Vascular Dementia Reflects the Vascular Disease Process
}

\author{
Karin Nilsson $^{a} \quad$ Lars Gustafson $^{a} \quad$ Björn Hultberg ${ }^{b}$ \\ a Department of Psychogeriatrics, Clinical Science, and bivision of Clinical Chemistry, \\ Department of Laboratory Medicine, Lund University Hospital, Lund, Sweden
}

\author{
Key Words \\ Cobalamin $\cdot$ Creatinine $\cdot$ Folate $\cdot$ Homocysteine $\cdot$ Depression · Vascular dementia $\cdot$ Vascular \\ disease
}

\section{Abstract}

Background: Patients with vascular dementia $(\mathrm{VaD})$ exhibit particularly elevated levels of plasma total homocysteine (tHcy) compared to patients with other psychogeriatric diseases. Methods: We investigated the main determinants (age, renal impairment, cobalamin/folate status and presence of extracerebral vascular disease) of plasma tHcy in 525 patients with VaD. Furthermore, 270 patients with depression were used as a reference group to reveal the potential specificity of elevated plasma tHcy in patients with VaD. Results: Elevated plasma tHcy levels in patients with $\mathrm{VaD}$ could only partly be attributed to cobalamin/folate deficiency or renal impairment. Plasma tHcy might also be related to the vascular disease process since patients with depression and vascular disease exhibited similar plasma tHcy levels to patients with VaD. Conclusion: Our findings suggest that elevated plasma tHcy might be a sensitive marker for the vascular disease process in patients with $\mathrm{VaD}$ and that the level also is a reflection of changes in the other main determinants of plasma tHcy.

Copyright (C) 2013 S. Karger AG, Basel 


\section{Introduction}

Apart from a dramatic impact on the personal life of patients and caregivers, dementia places a high burden on social and economic resources. With an ageing population, the prevalence of dementia will continue to rise in the coming decades. Since effective and specific treatments are lacking, prevention of dementia deserves high priority. Alzheimer's disease (AD) and vascular dementia (VaD) are the most prevalent forms of dementia, together accounting for up to $80 \%$ of all dementia cases [1]. The strict division between AD and VaD has faded with advancing research in neuropathology, neuroradiology and epidemiology, and the vascular component of AD has been increasingly recognized [2].

Elevated plasma homocysteine, decreased folate and low cobalamin serum levels have been shown to be associated with poor cognitive function, cognitive decline and dementia [3-5]. Plasma total homocysteine (tHcy) concentration is a function of a complex interaction between age and multiple genetic and environmental factors [6-8]. The most important factors are age, cobalamin/folate status and renal function [6-8]. Many clinical and epidemiological studies published during the last three decades have shown that even mild hyperhomocysteinemia is associated with vascular disease [6, 8-10]. Homocysteine metabolism might be compromised in elderly individuals in whom a low folate/cobalamin status is common [6]. The metabolism of folate and cobalamin is connected in one reaction where the remethylation of homocysteine to methionine is catalyzed by the cobalamin- and folatedependent enzyme methionine synthase $[6,8]$, and subsequently S-adenosylmethionine is formed. This metabolite is involved in numerous methylation reactions, involving proteins, phospholipids, DNA and neurotransmitter metabolism [6, 8]. Thus, elevated plasma tHcy levels are associated with low methylation potential, resulting in disturbed synthesis of lipids, nucleic acids and neurotransmitters as well as disturbed protein function and gene expression $[11,12]$. It has been suggested that a defect in the methylation processes is central to the neuropsychiatric manifestations of these vitamin deficiencies [11].

Elevated levels of plasma tHcy have been observed in both $\mathrm{AD}$ and $\mathrm{VaD}$, and in several studies [13-17] it has been shown that patients with VaD exhibited the highest plasma tHcy concentration. Although in most studies an increase in plasma tHcy in AD patients has been detected [18], our findings suggest that plasma tHcy is not primarily involved in the pathogenesis of $\mathrm{AD}$ but is rather a reflection of changes in the main determinants of plasma tHcy in $\mathrm{AD}$ patients $[19,20]$. In these studies, $\mathrm{AD}$ patients with normal cobalamin/folate status, normal serum creatinine and without vascular disease showed normal levels of plasma tHcy.

Currently, control of potential risk factors is believed to be the most effective method for preventing $\mathrm{VaD}$ [21]. Identification of modifiable risk factors for $\mathrm{VaD}$ is crucial for the development of effective treatment modalities. As plasma tHcy is particularly elevated in patients with $\mathrm{VaD}$, it may be one such risk factor for dementia [3-5]. We have previously investigated a large elderly population of psychogeriatric patients enrolled over 16 years (1990-2005) using essentially the same diagnostic criteria and biochemical parameters $[16,22-24]$. In this study, 525 patients with VaD were included. In addition, 270 patients with depression, with a similar age distribution to patients with $\mathrm{VaD}$, were included and used as a reference group to reveal the potential specificity of elevated plasma tHcy levels in patients with VaD. In the present study, we investigated plasma tHcy and its main determinants such as age, renal impairment, cobalamin/folate status and the presence of vascular disease in patients with $\mathrm{VaD}$ in order to elucidate the possible pathophysiological role of plasma tHcy in VaD. 


\section{Patients and Methods}

\section{Study Population}

The present study population consisted of 795 patients (318 males and 477 females, median age 81 years, 10 th-90th percentiles 70-88years) who were referred to the Department of Psychogeriatrics at the Lund University Hospital for diagnostic examination and treatment. The patients were consecutively enrolled during the period of 1990-2005. Patients on any kind of ongoing vitamin substitution were excluded from the study (15.2\% of the study population). The relative prevalence of patients with each diagnosis in the group of excluded patients did not significantly differ ( $\chi^{2}$ test) from that of the study group. The majority of the participants were living in their own homes, alone or with relatives. A total of 525 patients had VaD (228 males and 297 females) and 270 suffered from depression ( 90 males and 180 females). The clinical diagnosis was based on the DSM-IV criteria [25]. Furthermore, patients with VaD were diagnosed in accordance with the NINDS-AIREN criteria [26]. No patients with mixed vascular and Alzheimer-type dementia were included. The diagnoses were also based on neurological, somatic and laboratory investigations, psychometric testing, measurements of regional cerebral blood flow, electroencephalography and computerized tomography or magnetic resonance imaging as previously described [22, 27]. However, in 65 patients with VaD no brain imaging was available. Blood samples for the determination of the biochemical parameters were collected at the first visit to the Department of Psychogeriatrics. The study was approved by the Ethics Committee of the University of Lund. Informed consent to participate was given by all subjects (or relatives if the patients were unable to communicate).

The patients were divided into different groups according to the presence of a diagnosis and/or symptoms indicating vascular disease in their medical records. Patients with vascular disease included those with diagnoses/symptoms such as $\mathrm{VaD}$, cerebral infarction, transient ischaemic attacks, myocardial infarction, angina pectoris, peripheral vascular disease, atrial fibrillation and hypertension.

Furthermore, 147 apparently healthy subjects ( 74 males and 73 females, median age 75 years, 10th-90th percentiles 45-85 years) served as an age-matched control group for plasma thcy levels. All control subjects exhibited normal levels (see below) of serum cobalamin, serum folate and serum creatinine. They had no history of cardiovascular disease and were taking no vitamin supplements according to a health assessment questionnaire.

Assays

Blood samples for homocysteine determination were collected in evacuated tubes containing EDTA at about 8 a.m. after an overnight fast and centrifuged within $15 \mathrm{~min}$ at $3,000 \mathrm{~g}$ for $5 \mathrm{~min}$. The plasma was stored at $-20^{\circ} \mathrm{C}$ until analysis. Plasma tHcy was measured with high-performance chromatography after reduction of disulphide bonds with dithiothreitol and deproteinization with sulphosalicylic acid as previously described [28].

Serum cobalamin as well as serum and blood folate levels were determined by competitive protein-binding assays at the Department of Clinical Chemistry, Lund University Hospital. For determination of serum cobalamin, we used the AutoDELFIA B12 kit (Wallac Oy, Turku, Finland) and an immunoassay from Roche Diagnostics (Modular Analytics E 170; Roche Diagnostics, Basel, Switzerland), which exhibited equivalent levels of serum cobalamin. Blood folate was determined with a Dualcount Solid Phase No Boil Assay (Diagnostic Products Corp., Los Angeles, Calif., USA) and serum folate with an immunoassay from Roche Diagnostics (Modular Analytics E 170, Roche Diagnostics, Basel, Switzerland). Blood folate was replaced by serum folate in 1998 for the assay of folate status. Thus, 481 patients were analyzed with blood folate and 262 with serum folate. For cobalamin in serum, we used a reference interval of $150-650 \mathrm{pmol} / \mathrm{l}[22,27]$. According to the manufacturer, the reference interval for blood 
Table 1. Plasma tHcy in patients older and younger than 75 years with normal cobalamin/folate status and normal level of serum creatinine (normal conditions) and with cobalamin/folate deficiency and elevated serum creatinine (pathological conditions) compared to controls (all with normal conditions)

\begin{tabular}{|c|c|c|c|c|}
\hline & \multicolumn{2}{|l|}{$<75$ years } & \multicolumn{2}{|l|}{ >75 years } \\
\hline & normal & pathological & normal & pathological \\
\hline $\mathrm{VaD}$ & $\begin{array}{l}13.3[9.5-21.8]^{* *} \\
(n=45)\end{array}$ & $\begin{array}{l}18.4[10.9-33.9]^{* * *} \\
(\mathrm{n}=44)^{\mathrm{a}}\end{array}$ & $\begin{array}{l}17.4[12.2-25.9]^{* * *} \\
(\mathrm{n}=190)\end{array}$ & $\begin{array}{l}21.6[14.2-35.8]^{* * *} \\
(\mathrm{n}=228)^{\text {aa }}\end{array}$ \\
\hline Depression & $\begin{array}{l}12.3[8-19.7] \\
(n=57)\end{array}$ & $\begin{array}{l}20.9[14.3-27.3]^{*} \\
(\mathrm{n}=6)^{\mathrm{a}}\end{array}$ & $\begin{array}{l}15.6[10.7-24.3]^{* *} \\
(\mathrm{n}=104)\end{array}$ & $\begin{array}{l}20.5[12.0-32.5]^{* * *} \\
(\mathrm{n}=84)^{\text {aa }}\end{array}$ \\
\hline Controls & $\begin{array}{l}12.1[8.9-16.9] \\
(n=60)\end{array}$ & & $\begin{array}{l}13.4[10.2-19.2] \\
(n=87)\end{array}$ & \\
\hline
\end{tabular}

Values are medians with 10th-90th percentiles in brackets.

$* \mathrm{p}<0.05 ;{ }^{* *} \mathrm{p}<0.01 ;{ }^{* *} \mathrm{p}<0.001$ : patients are compared to controls.

${ }^{a} \mathrm{p}<0.05$; ${ }^{\text {aa }} \mathrm{p}<0.01$ : patients with pathological conditions are compared to respective patients with normal conditions.

folate is $180-725 \mathrm{nmol} / \mathrm{l}$ and that for serum folate 7-39 $\mathrm{nmol} / \mathrm{l}$ [29]. Therefore, the lower limit for blood folate had been changed somewhat in the present study from the $125 \mathrm{nmol} / \mathrm{l}$ used in previous studies [22,27] to $180 \mathrm{nmol} / \mathrm{l}$, which is in accordance with the manufacturer's recommendation and also more in agreement with the lower limit for folate in serum [29]. Serum creatinine was determined with an enzymatic routine method at the Department of Clinical Chemistry. The upper reference limits for serum creatinine are $105 \mu \mathrm{mol} / \mathrm{l}$ for men and $90 \mu \mathrm{mol} / \mathrm{l}$ for women $[22,27]$.

\section{Statistics}

The results are presented as medians and 10th-90th percentiles. The following twotailed tests at the $5 \%$ level of significance were used to evaluate the study: to avoid the possibility of skewed distributions, nonparametric methods were used. The Mann-Whitney U test was used in the case of two independent samples. Stepwise multiple regression analysis was performed to evaluate the predictors of plasma tHcy. The $\chi^{2}$ test was used for comparison of the percentage of vascular disease.

\section{Results}

The Relation of Plasma tHcy Level to Cobalamin/Folate Deficiencies and Elevated Serum Creatinine in Different Age Groups

Patients and controls were divided into two age groups, above and below 75 years (table 1). Patients in each age group were further divided into two groups: one group with normal folate/cobalamin status and normal serum creatinine (normal conditions) and another group with deficiencies of folate/cobalamin status and/or elevated serum creatinine (pathological conditions). Patients with VaD showed significantly higher plasma tHcy concentration in all groups compared to healthy subjects. Patients with depression showed an increased plasma tHcy concentration in all groups except for patients with normal conditions younger than 75 years, although the younger patients with pathological conditions 
Table 2. Patients with $\mathrm{VaD}$ and depression older than 75 years and with normal conditions divided into two groups with plasma tHcy above and below the median

\begin{tabular}{|c|c|c|c|c|c|c|c|c|}
\hline & $\mathrm{n}$ & $\begin{array}{l}\text { Age } \\
\text { years }\end{array}$ & $\begin{array}{l}\text { Serum } \\
\text { cobalamin } \\
\text { pmol/l }\end{array}$ & $\begin{array}{l}\text { Plasma tHcy } \\
\mu \mathrm{mol} / \mathrm{l}\end{array}$ & $\begin{array}{l}\text { Serum } \\
\text { folate } \\
\text { nmol/l }\end{array}$ & $\begin{array}{l}\text { Blood } \\
\text { folate } \\
\text { nmol/l }\end{array}$ & $\begin{array}{l}\text { Serum } \\
\text { creatinine } \\
\mu \text { mol/l }\end{array}$ & $\begin{array}{l}\text { Vascular } \\
\text { disease } \\
\%\end{array}$ \\
\hline $\begin{array}{l}\text { VaD patients with tHcy below } \\
\text { median }(17.4 \mu \mathrm{mol} / \mathrm{l})\end{array}$ & 96 & $82[76-88]$ & 285 [198-504] & $13.8[10.3-16.4]$ & $\begin{array}{l}11[7.7-26.8] \\
(\mathrm{n}=30)\end{array}$ & $\begin{array}{l}312[210-547] \\
(n=66)\end{array}$ & 72 [53-91] & 100 \\
\hline $\begin{array}{l}\text { VaD patients with tHcy above } \\
\text { median }(17.4 \mu \mathrm{mol} / \mathrm{l})\end{array}$ & 94 & 83 [76-90] & $257[159-430]^{* *}$ & $21.4[18-29.3]^{* * *}$ & $\begin{array}{l}10[7.8-14] \\
(\mathrm{n}=33)\end{array}$ & $246[193-407]^{* *}$ & $81[63-98]^{* * *}$ & 100 \\
\hline $\begin{array}{l}\text { Patients with depression and } \\
\text { tHcy below median }(15.6 \mu \mathrm{mol} / \mathrm{l})\end{array}$ & 53 & 80 [75-89] & 276 [186-593] & $12.1[9.5-15.1]$ & $\begin{array}{l}17[9.2-38] \\
(n=19)\end{array}$ & $\begin{array}{l}357[228-643] \\
(n=34)\end{array}$ & 71 [49-89] & 36 \\
\hline $\begin{array}{l}\text { Patients with depression and } \\
\text { tHcy above median }(15.6 \mu \mathrm{mol} / \mathrm{l})\end{array}$ & 51 & 82 [77-87] & 255 [172-473] & $20[15.9-26.4]^{* * *}$ & $\begin{array}{l}7.4[7-8.5]^{* * *} \\
(\mathrm{n}=4)\end{array}$ & $\begin{array}{l}254[196-366]^{* * *} \\
(\mathrm{n}=47)\end{array}$ & 72 [49-96] & 41 \\
\hline
\end{tabular}

Values are medians with 10th-90th percentiles in brackets.

${ }^{* *} \mathrm{p}<0.01 ;{ }^{* * *} \mathrm{p}<0.001$ : patients with plasma tHcy below the median are compared to those above the median.

showed only a slight significant increase in plasma tHcy. Furthermore, both VaD patients and patients with depression younger and older than 75 years with pathological conditions showed a significant increase compared to the respective patient groups with normal conditions.

Patients older than 75 years with $\mathrm{VaD}$ and depression and with normal cobalamin/folate status and normal levels of serum creatinine were divided into two groups according to their plasma tHcy level (above or below the median) (table 2). Patients with VaD and plasma tHcy above the median $(17.4 \mu \mathrm{mol} / \mathrm{l})$ showed lower levels of serum cobalamin, blood folate and elevated serum creatinine than those with plasma tHcy below the median, whereas age and serum folate were unchanged. Patients with depression and plasma tHcy above the median $(15.6 \mu \mathrm{mol} / \mathrm{l})$ presented lower levels of serum and blood folate than those with plasma tHcy below the median, whereas age, serum cobalamin, serum creatinine and presence of vascular disease were unchanged.

Multiple regression analyses in all VaD patients with plasma tHcy as the dependent variable and age, gender, serum cobalamin, serum creatinine, folate deficiency (yes/no) and presence of extracerebral vascular disease (yes/no) as independent variables revealed that serum creatinine, folate deficiency, serum cobalamin and gender significantly predicted plasma tHcy level. The predictive value $\left(\mathrm{R}^{2}\right.$ adjusted, expressed as percentage) of these four parameters was $23.8 \%$ (creatinine $10.3 \%$, folate deficiency $8.8 \%$, cobalamin $3.9 \%$ and gender $0.8 \%$ ).

\section{Percentage of Cobalamin/Folate Deficiencies and Elevated Serum Creatinine}

Low levels of cobalamin and/or folate in VaD patients younger than 75 years were $32.3 \%$ and in patients older than 75 years $26.9 \%$, whereas patients with depression exhibited a lower percentage of lower levels (5.9\% below and $17.1 \%$ above 75 years) (table 3). Elevated levels of serum creatinine were more frequent $(22 \%)$ in patients with VaD (11\% below and $25 \%$ above 75 years) compared to patients with depression (13\%; $2.8 \%$ below and $17.6 \%$ above 75 years).

\section{Plasma tHcy Concentration in Patients with VaD and Depression in Relation to Vascular Disease}

Table 4 shows the plasma tHcy concentration in all VaD patients and in VaD patients with and without extracerebral vascular disease. Patients with concomitant extracerebral vascular disease presented with higher blood folate and a lower level of serum creatinine than patients without extracerebral vascular disease. Despite these findings, plasma tHcy showed a 
Table 3. Percentage of cobalamin/folate deficiency and elevated serum creatinine in patients older and younger than 75 years

\begin{tabular}{llll}
\hline & Patients $<75$ years & Patients $>75$ years & All patients \\
\hline VaD & & & 525 \\
Patients, n & 89 & 418 & 9.5 \\
Cobalamin deficiency, \% & 9.0 & 9.8 & 18.1 \\
Folate deficiency, \% & 23.3 & 17.1 & 4.0 \\
Cobalamin/folate deficiency, \% & 6.0 & 3.0 & 22.0 \\
Elevated creatinine, \% & 11.0 & 25 & \\
\hline Depression & & & 270 \\
Patients, $\mathrm{n}$ & 71 & 188 & 5.9 \\
Cobalamin deficiency, \% & 2.8 & 7.0 & 8.1 \\
Folate deficiency, \% & 3.1 & 10.1 & 6.3 \\
Cobalamin/folate deficiency, \% & - & 7.1 & 13.0 \\
Elevated creatinine, \% & 2.8 & 17.6 & \\
\hline
\end{tabular}

Table 4. Age, plasma tHcy, serum cobalamin, blood or serum folate, and serum creatinine in all VaD patients and in VaD patients with and without extracerebral vascular disease and in patients with depression with and without vascular disease

\begin{tabular}{|c|c|c|c|c|c|c|c|}
\hline & $\mathrm{n}$ & $\begin{array}{l}\text { Age } \\
\text { years }\end{array}$ & $\begin{array}{l}\text { Plasma tHcy } \\
\mu \mathrm{mol} / \mathrm{l}\end{array}$ & $\begin{array}{l}\text { Serum } \\
\text { cobalamin } \\
\text { pmol/l }\end{array}$ & $\begin{array}{l}\text { Serum folate } \\
\mathrm{nmol} / \mathrm{l}\end{array}$ & $\begin{array}{l}\text { Blood folate } \\
\text { nmol/l }\end{array}$ & $\begin{array}{l}\text { Serum } \\
\text { creatinine } \\
\mu \mathrm{mol} / \mathrm{l}\end{array}$ \\
\hline \multicolumn{8}{|l|}{$V a D$} \\
\hline All patients & 525 & $82[72-89]$ & $18.9[12-30.5]$ & 258 [145 - 479] & $9.9[5.2-18]$ & $256[156-486]$ & $83[59-126$ \\
\hline $\begin{array}{l}\text { Cerebral and extracerebral } \\
\text { vascular disease }\end{array}$ & 344 & $82[72-89]$ & $19.2[12.1-30.5]$ & $270[156-474]$ & $\begin{array}{l}9.1[5.0-17.7] \\
(n=132)\end{array}$ & $\begin{array}{l}270[161-532] \\
(n=204)\end{array}$ & 83 [59-126] \\
\hline Cerebral vascular disease & 116 & $82[72-89]$ & 18.3 [12.4-31.7] & 265 [153-499] & $\begin{array}{l}10[5.7-25] \\
(n=49)\end{array}$ & $\begin{array}{l}245[158-451]^{*} \\
(\mathrm{n}=63)\end{array}$ & $89[63-127]^{*}$ \\
\hline \multicolumn{8}{|l|}{ Depression } \\
\hline Vascular disease & 110 & $81[71-88]$ & $17.5[10.7-26.3]^{\mathrm{b}}$ & $301[158-539]^{a-c}$ & $\begin{array}{l}14[6.3-26.4] \\
(\mathrm{n}=25)^{\mathrm{aa}, \mathrm{bb}, \mathrm{c}}\end{array}$ & $\begin{array}{l}288[185-632] \\
(\mathrm{n}=79)^{\mathrm{a}-\mathrm{c}}\end{array}$ & 83 [55-131] \\
\hline No vascular disease & 100 & $78[53-86]^{* * *}$ & $14.3[9.4-24.3]^{* * *}$ & $282[167-539]$ & $\begin{array}{l}15.5[6.7-30.3] \\
(n=38)\end{array}$ & $\begin{array}{l}284[182-589] \\
(n=57)\end{array}$ & $74[52-98]^{* *}$ \\
\hline
\end{tabular}

Values are medians with 10 th-90th percentiles in brackets.

$* \mathrm{p}<0.05 ;{ }^{* *} \mathrm{p}<0.01 ;^{* * *} \mathrm{p}<0.001$ : VaD patients without extracerebral vascular disease $(\mathrm{n}=116)$ are compared to patients with concomitant extracerebral vascular disease $(n=344)$. Patients with depression and vascular disease $(n=110)$ are compared to patients without vascular disease $(n=100)$.

${ }^{\mathrm{a}} \mathrm{p}<0.05$; ${ }^{\text {aa }} \mathrm{p}<0.01$ : all patients with $\mathrm{VaD}(\mathrm{n}=525)$ are compared to patients with depression and vascular disease. ${ }^{\mathrm{b}} \mathrm{p}<0.05$; ${ }^{\text {bb }} \mathrm{p}<0.01$ : VaD patients with concomitant extracerebral vascular disease $(n=344)$ are compared to patients with depression and vascular disease. ${ }^{c} p<0.05$ : VaD patients without concomitant extracerebral vascular disease $(n=116)$ are compared to patients with depression and vascular disease.

tendency towards higher levels, although not significant, in patients with concomitant extracerebral vascular disease.

Patients with depression and vascular disease were somewhat older and exhibited higher levels of plasma tHcy and serum creatinine than patients without vascular disease.

There was no significant increase in plasma tHcy neither in all patients with VaD nor in VaD patients without concomitant extracerebral vascular disease, although cobalamin and folate levels were significantly higher in patients with depression and vascular disease compared to the different groups of $\mathrm{VaD}$ patients. In VaD patients with concomitant extracerebral vascular disease, a slightly increased plasma tHcy concentration was observed compared to patients with depression and vascular disease. However, this significant difference disappeared after the exclusion of patients with cobalamin/folate deficiency and/ or elevated serum creatinine in these two groups (data not shown). 


\section{Discussion}

Elevated plasma tHcy levels in patients with VaD could partly be attributed to cobalamin/ folate deficiency or renal impairment since we observed an increased level of plasma tHcy in patients with pathological conditions compared to patients with normal conditions. Furthermore, among VaD patients older than 75 years with cobalamin, folate and creatinine within reference ranges (normal conditions), lower levels of serum cobalamin, blood folate and higher levels of serum creatinine were observed in the patients with plasma tHcy above the median compared to the patients with plasma tHcy below the median. Thus, the increased level of plasma tHcy in the patients with normal conditions was associated with lower levels of cobalamin/folate and/or elevated serum creatinine, although these parameters were within the reference range in these patients. Likewise, a multiple regression analysis in all VaD patients showed mainly the importance of serum creatinine and folate deficiency and, to a lesser degree, of serum cobalamin for the prediction of plasma tHcy levels. The relatively limited effect on the plasma tHcy level of dividing patients older than 75 years into two groups with normal and pathological conditions might be related to the fact that there is no definitive test to reveal marginal cobalamin/folate deficiency. Therefore, it is possible that patients with marginal cobalamin/folate deficiencies might still remain in the group with normal conditions. Likewise, serum creatinine is not a perfect measure of renal function since its concentration is not only related to renal function but also to muscle mass [30].

An increasing prevalence of cobalamin and folate deficiencies with age has been reported in elderly subjects [31]. In the United Kingdom, cobalamin deficiency was observed in about $5 \%$ of an elderly population aged 65-74 years and in $10 \%$ of subjects aged over 75 years. Folate deficiency was also common in older subjects, and its prevalence was similar to that of cobalamin deficiencies, but only about $10 \%$ of subjects with low cobalamin levels also had low folate levels [31]. In the present study, the frequency of low levels of cobalamin and/or folate in $\mathrm{VaD}$ patients was $32.3 \%$ before the age of 75 years and $26.9 \%$ after the age of 75 years, whereas patients with depression exhibited a lower percentage of lower levels $(5.9 \%$ before and $17.1 \%$ after the age of 75 years). The total percentage of cobalamin/folate deficiency is, however, probably higher in both groups of patients since about $15 \%$ of all referred patients were excluded from the study due to treatment for vitamin deficiency (see Patients and Methods). Therefore, cobalamin/folate deficiency is probably more frequent in VaD patients than in normal elderly subjects. Furthermore, patients with VaD showed a high frequency of elevated serum creatinine levels (22\%) compared to patients with depression (13\%). Since the deterioration of renal function with age is well known [30], it is important to stress that the patients with depression were in the same age range as the patients with VaD. Thus, our study provides evidence that cobalamin/folate status and renal function are important for elevated plasma tHcy in patients with $\mathrm{VaD}$. It is notable that a substantial share (about $40 \%$ ) of the patients with VaD had cobalamin/folate deficiency and/or renal insufficiency. These results are in agreement with those of previous studies in which levels of tHcy, folate, cobalamin and creatinine were measured in VaD patients [13-17]. The majority of the studies agree that plasma tHcy is increased and that the higher plasma tHcy level is associated with lower levels of folate, cobalamin and elevated creatinine. This assumption is also supported by the findings of the present study where multiple regression analysis showed that folate/cobalamin deficiency and/or creatinine significantly predicted plasma tHcy in $\mathrm{VaD}$ patients. Even within the reference range, folate/cobalamin and creatinine showed an association with plasma tHcy.

Elevated plasma homocysteine, decreased folate and low cobalamin serum levels are associated with poor cognitive function, cognitive decline and dementia [3]. There is a substantial body of literature linking homocysteine, folic acid and cobalamin with cognitive 
decline. Despite this association, there is to date no clear evidence from randomized controlled trials that supplementation with folic acid and cobalamin improves cognitive decline or dementia, even though it may normalize plasma tHcy levels as recently reviewed [3, 32]. However, these studies are heterogeneous in terms of the population studied, the definition of cobalamin or folate deficiency, the assessment and definition of cognitive impairment, type of dementia and study design.

Patients with VaD without extracerebral vascular disease showed similar levels of plasma tHcy, despite lower levels of blood folate and higher levels of serum creatinine, than patients with VaD and a concomitant extracerebral vascular disease. Furthermore, patients with depression and vascular disease exhibited similar plasma tHcy levels to all patients with $\mathrm{VaD}$ and VaD patients without concomitant extracerebral vascular disease, although cobalamin and folate levels were significantly higher in patients with depression and vascular disease than in VaD patients. Only VaD patients with concomitant extracerebral vascular disease showed a slightly increased plasma tHcy level compared to patients with depression and vascular disease (which disappeared after the exclusion of patients with cobalamin/folate deficiency and/or elevated serum creatinine in these two groups). These findings therefore imply that the particular increase in plasma tHcy in patients with VaD compared to AD patients [13-17] might be related to the vascular disease process in VaD patients.

In conclusion, the main determinants of plasma tHcy, cobalamin/folate deficiency and/ or renal impairment, may partially explain the elevated levels of plasma tHcy in VaD patients observed in many studies [13-17]. The findings of the present study also imply that the particular increase in plasma tHcy in patients with $\mathrm{VaD}$ might be related to the vascular disease process in these patients. Plasma tHcy, however, is not a specific risk factor for VaD since patients with depression and vascular disease exhibited a similar increase in plasma tHcy to patients with VaD. Ascertaining plasma tHcy and its main determinants might be valuable in patients with $\mathrm{VaD}$ to judge the severity of the vascular disease process. In this context, it is interesting that we have recently observed that plasma tHcy is a significant predictor of mortality in patients with VaD [24].

\section{Acknowledgements}

This work was supported by grants from the Swedish Medical Research Council (grant No. 3950), the Alzheimer Foundation Sweden, the Sjöbring Foundation, the Swedish Heart Lung Foundation, the Trolle-Wachtmeister Foundation for Medical Research, the Albert Påhlsson Foundation and the County Council of Malmöhus.

\section{References}

1 Fratiglioni LM, Launer LJ, Andersen KM, et al: Incidence of dementia and major subtypes in Europe: a collaborative study of population-based cohorts. Neurology 2000;54:S10-S15.

- 2 Grammas P: Neurovascular dysfunction, inflammation and endothelial activation: implications for the pathogenesis of Alzheimer's disease. J Neuroinflammation 2011;8:26-38.

- 3 Vogel T, Dali-Youcef N, Kaltenbach G, Andres E: Homocysteine, vitamin B12, folate and cognitive functions: a systematic and critical review of the literature. Int J Clin Pract 2009;63:1061-1067.

- 4 Seshadri S, Beiser A, Selhub J, Jacques PF, Rosenberg IH, D’Agostino RB, Wilson PW, Wolf PA: Plasma homocysteine as a risk factor for dementia and Alzheimer's disease. N Engl J Med 2002;346:476-483.

5 Seshadri S: Elevated plasma homocysteine levels: risk factor or risk marker for the development of dementia and Alzheimer's disease? J Alzheimers Dis 2006;9:393-398.

6 Refsum H, Smith DA, Ueland PM, Nexö E, Clarke R, McPartlin J, Johnston C, Engbaek F, Schneede J, McPartlin C, Scott JM: Facts and recommendations about total homocysteine determinations: an expert opinion. Clin Chem 2004;50:3-32. 
Nilsson et al.: Elevated Plasma Homocysteine Level in Vascular Dementia Reflects the

Vascular Disease Process

7 Brattström L, Lindgren A, Israelsson B, Andersson A, Hultberg B: Homocysteine and cysteine: determinants of plasma levels in middle-aged and elderly subjects. J Intern Med 1994;236:633-641.

- 8 Castro R, Rivera I, Blom HJ, Jakobs C, Tavares de Almeida I: Homocysteine metabolism, hyperhomocysteinaemia and vascular disease: an overview. J Inherit Metab Dis 2006;29:3-20.

- 9 Wald DS, Law M, Morris JK: Homocysteine and cardiovascular disease: evidence on causality from a metaanalysis. BMJ 2002;325:1202-1209.

10 Homocysteine Studies Collaboration: Homocysteine and risk of ischemic heart disease and stroke: a metaanalysis. JAMA 2002;288:2015-2022.

11 Obeid R, McCaddon A, Herrmann W: The role of hyperhomocysteinemia and B-vitamin deficiency in neurological and psychiatric diseases. Clin Chem Lab Med 2007;45:1590-1606.

12 Coppedè F: One-carbon metabolism and Alzheimer's disease: focus on epigenetics. Curr Genomics 2010;11: 246-260.

13 Clarke R, Smith AD, Jobst KA, Refsum H, Sutton L, Ueland PM: Folate, vitamin B12, and serum total homocysteine levels in confirmed Alzheimer disease. Arch Neurol 1998;55:1449-1455.

14 Mcllroy SP, Dynan KB, Lawson JT, Patterson CC, Passmore AP: Moderately elevated plasma homocysteine, methylenetetrahydrofolate reductase genotype and risk for stroke, vascular dementia and Alzheimer disease in Northern Ireland. Stroke 2002;33:2351-2356.

15 Quadri P, Fragiacomo C, Pezzati R, Zanda E, Forloni G, Tettamanti M, Lucca U: Homocysteine, folate and vitamin B-12 in mild cognitive impairment, Alzheimer's disease and vascular dementia. Am J Clin Nutr 2004;80:114122.

16 Nilsson K, Gustafson L, Hultberg B: Elevated plasma homocysteine concentration in elderly patients with mental illness is mainly related to the presence of vascular disease and not the diagnosis. Dement Geriatr Cogn Disord 2007;24:162-168.

$\checkmark 17$ Ho RC, Cheung MW, Fu E, Win HH, Zaw MH, Ng A, Mak A: Is high homocysteine level a risk factor for cognitive decline in the elderly? A systematic review, meta-analysis, and meta-regression. Am J Geriatr Psychiatry 2011; 19:607-617.

18 Zhuo JM, Wang H, Pratico D: Is hyperhomocysteinemia an (AD) risk factor and AD marker, or neither? Trends Pharmacol Sci 2011;32:562-571.

19 Nilsson K, Gustafson L, Hultberg B: Relation between plasma homocysteine and Alzheimer's disease. Dement Geriatr Cogn Disord 2002;14:7-12.

20 Nilsson K, Gustafson L, Hultberg B: Plasma homocysteine concentration relates to the severity but not to the duration of Alzheimer's disease. Dement Geriatr Cogn Disord 2004;19:666-672.

21 Perez L, Heim L, Sherzai A, Jaceldo-Siegl K, Sherzai A: Nutrition and vascular dementia. J Nutr Health Aging 2012;16:319-324.

22 Nilsson K, Gustafson L, Hultberg B: Plasma homocysteine, cobalamin/folate status, and vascular disease in a large population of psychogeriatric patients. Dement Geriatr Cogn Disord 2006;22:358-366.

-23 Nilsson K, Gustafson L, Hultberg B: Plasma homocysteine levels and different forms of vascular disease in patients with dementia and other psychogeriatric diseases. Dement Geriatr Cogn Disord 2009;27:88-95.

24 Nilsson K, Gustafson L, Hultberg B: Survival in a large population of patients with dementia and other forms of psychogeriatric diseases. Dement Geriatr Cogn Disord 2011;32:342-350.

25 American Psychiatric Association: DSM IV: Diagnostic and Statistical Manual of Mental Disorders, ed 4. Washington, American Psychiatric Association, 1994.

26 Román GC, Tatemichi TK, Erkinjuntti T, Cummings JL., Masdeu JC, et al: Vascular dementia: diagnostic criteria for research studies. Report of the NINDS-AIREN International Workshop. Neurology 1993;43:250-260.

-27 Nilsson K, Gustafson L, Fäldt R, Andersson A, Brattström L, Lindgren A, Israelsson B, Hultberg B: Hyperhomocysteinaemia - a common finding in a psychogeriatric population. Eur J Clin Invest 1996;26:853-859.

-28 Andersson A, Isaksson A, Brattström L, Hultberg B: Homocysteine and other thiols determined in plasma by HPLC and thiol-specific postcolumn derivatization. Clin Chem 1993;39:1590-1597.

29 Hultberg B, Nilsson K, Isaksson A, Gustafson L: Folate deficiency is a common finding in psychogeriatric patients. Aging Clin Exp Res 2002;14:479-484.

30 Norlund L, Grubb A, Fex G, Leksell H, Nilsson J-E, Schenk H, Hultberg B: The increase of plasma homocysteine concentration with age is partly due to the deterioration of renal function as determined by plasma cystatin C. Clin Chem Lab Med 1998;36:175-178.

-31 Clarke R, Grimley Evans J, Schneede J, Nexo E, Bates C, Fletcher A, Prentice A, Johnston C, Ueland PM, Refsum H, Sherliker P, Birks J, Whitlock G, Breeze E, Scott JM: Vitamin B12 and folate deficiency in later life. Age Ageing 2004;33:34-41.

32 Wald DS, Kasturiatne A, Simmonds M: Effects of folic acid, with or without other B vitamins, on cognitive decline: meta-analysis of randomized trials. Am J Med 2010;123:1320-1326. 